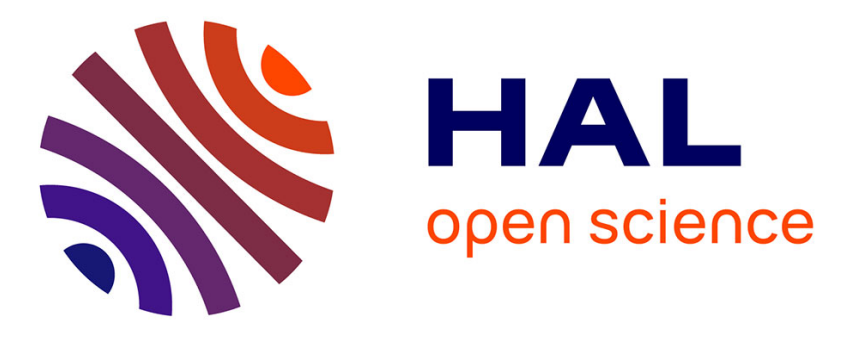

\title{
Extreme events in optics: Challenges of the MANUREVA project
}

John M. Dudley, Christophe Finot, Guy Millot, Josselin Garnier, Goëry Genty, Dmitry Agafontsev, Frédéric Dias

\section{- To cite this version:}

John M. Dudley, Christophe Finot, Guy Millot, Josselin Garnier, Goëry Genty, et al.. Extreme events in optics: Challenges of the MANUREVA project. The European Physical Journal. Special Topics, 2010, 185 (1), pp.125-133. 10.1140/epjst/e2010-01243-x . hal-00510503

\section{HAL Id: hal-00510503 \\ https://hal.science/hal-00510503}

Submitted on 19 Aug 2010

HAL is a multi-disciplinary open access archive for the deposit and dissemination of scientific research documents, whether they are published or not. The documents may come from teaching and research institutions in France or abroad, or from public or private research centers.
L'archive ouverte pluridisciplinaire HAL, est destinée au dépôt et à la diffusion de documents scientifiques de niveau recherche, publiés ou non, émanant des établissements d'enseignement et de recherche français ou étrangers, des laboratoires publics ou privés. 


\title{
Extreme events in optics : challenges of the MANUREVA project
}

\author{
J. M. Dudley, a, C. Finot ${ }^{2}$, G. Millot ${ }^{2}$, J. Garnier ${ }^{3}$, G. Genty ${ }^{4}$, D. Agafontsev ${ }^{5}$, and F. Dias ${ }^{5}$ \\ 1 Departément d'Optique P. M. Duffieux, Institut FEMTO-ST, UMR 6174 CNRS-Université de Franche- \\ Comté, 25030 Besançon, France \\ 2 Institut Carnot de Bourgogne, UMR 5209 CNRS-Université de Bourgogne, 9 Av. A. Savary, Dijon, \\ France \\ 3 Laboratoire de Probabilités et Modèles Aléatoires and Laboratoire Jacques-Louis Lions, Université \\ Paris VII, 75205 Paris Cedex 13, France \\ 4 Tampere University of Technology, Optics Laboratory, FI-33101 Tampere, Finland \\ 5 Centre de Mathématique et de Leurs Applications (CMLA), ENS Cachan, France
}

\begin{abstract}
In this contribution we describe and discuss a series of challenges and questions relating to understanding extreme wave phenomena in optics. Many aspects of these questions are being studied in the framework of the MANUREVA project: a multidisciplinary consortium aiming to carry out mathematical, numerical and experimental studies in this field. The central motivation of this work is the 2007 results from optical physics [D. Solli et al. Nature 450, 1054-1057 (2007)] that showed how a fibre-optical system can generate large amplitude extreme wave events with similar statistical properties to the infamous hydrodynamic rogue waves on the surface of the ocean. We review our recent work in this area, and discuss how this observation may open the possibility for an optical system to be used to directly study both the dynamics and statistics of extreme-value processes, a potential advance comparable to the introduction of optical systems to study chaos in the 1970s.
\end{abstract}

\section{Introduction}

Extreme value phenomena have dramatic impact in many fields in the physical and social sciences, and are thus the subject of intense international research [1]. The potential of a given system to generate extreme events reflects the complexity of the underlying dynamics, and a central challenge in understanding extreme events is to develop rigorous models linking these dynamics and the associated statistical behaviour. The scientific study of extreme value phenomena, however, has been significantly hampered in two ways: (i) the intrinsic scarcity of the events under study and (ii) the fact that such events are often of most interest when they appear in environments (e.g. the ocean) where measurements are difficult. These unavoidable problems have led to an absence of extensive data sets generated under controlled conditions, resulting in difficulties in studying their generation mechanisms and statistical properties in a quantitative manner.

In this context, however, recent research from the domain of optical physics published in Nature in late 2007 has attracted significant interest [2]. In particular, this research has shown that a convenient laboratory-based optical fibre system can generate extreme value events with similar statistical properties to the large amplitude hydrodynamic rogue waves observed on the

\footnotetext{
a e-mail: john.dudley@univ-fcomte.fr
} 
surface of the ocean. In fact, although analogies between linear and nonlinear wave propagation in hydrodynamics and optics have been known since the 1960's (see Ref. [3] for background), the significance of the 2007 Nature article is that it reported a novel experimental technique for directly quantifying the extreme statistical fluctuations that occur during the highly nonlinear process of white light supercontinuum generation.

These 2007 results have since motivated tremendous interest in studying the ways in which an optical system can be used to directly and conveniently study extreme-value processes. Studying the links between nonlinear optics, extreme-value theory and hydrodynamic wave formation, however, necessarily requires a multidisciplinary approach and in this context, the MANUREVA consortium was established ${ }^{1}$. Initially a French national collaboration, many different groups are now working with the original partners, and activity in this field has also spread very rapidly within the international physics and mathematics communities (this Special Issue is an example!) The aim of this article is to present an overview of the current research aims of this project which to some degree reflect the wider challenges and questions of the field of optical rogue wave physics. Our primary aim is to introduce a number of key ideas and references from related areas of nonlinear physics that may prove useful in moving progress forward in this exciting and rapidly-developing field.

\section{Rogue Waves in Hydrodynamics}

Although our aim here is to focus primarily on the open questions relevant to extreme events in optics, it is useful here to present a short review of the general context of the field of oceanic rogue waves. Oceanic rogue (or freak) waves can be conveniently defined as statistically-rare and spontaneous surface waves that reach extreme amplitudes, and which have been held responsible for numerous marine disasters ranging from the sudden sinking of seagoing ships to damage to open sea oil platforms. There are of course more quantitative and complete criteria that are used to define ocean rogue waves, but is not our aim here to summarise the vast literature in this field; the reader is referred rather to several excellent monographs and recent conference proceedings [4-6].

For our purposes in this article, it is sufficient to restate the obvious fact that the formation and propagation of surface waves on the ocean is influenced by a number of driving processes, including local winds and weather systems, rapid ocean currents, and the effect of both seabed and coastal topography. Both linear and non-linear processes have been studied in order to understand the physics of rogue wave formation, and the excitation of waves of unusually large amplitude has been shown to arise from a number of different mechanisms. Amongst those that have received particular attention are: spatial focussing due to refraction in the presence of varying topography or currents; directional focussing of multiple wave trains; the dispersive focussing of a chirped wave train; and nonlinear processes arising from the exponential amplification of random surface noise through the Benjamin-Feir/Bespalov-Talanov modulation instability (MI) [7,8], or the development of high amplitude coherent structures from solitonic or quasi-solitonic wave turbulence. It is the possibility that rogue events are generated from such nonlinear mechanisms that has motivated the optics community to pay such attention.

The degree to which the different mechanisms act independently or in combination is, however, an open question, and it is particularly unclear whether nonlinear processes are at the origin of all rogue wave phenomena, or only involved in the focussing stage of extreme sea states that have been initiated by some other linear process [9]. Resolving this particular question is crucial to a full understanding of rogue wave physics, yet doing so requires a detailed and coupled investigation into the wave dynamics and the accompanying statistics.

Unfortunately, such a study is extremely difficult to carry out in the natural marine environment, and even experiments in wave tanks provide only limited insight into the results expected under realistic conditions. Detailed and realistic simulations of real ocean events are

\footnotetext{
1 The long title is: "Mathematical modelling and experiments studying nonlinear instabilities, rogue waves and extreme phenomena"
} 
only just beginning to appear [10] and there remain a number of open questions relating to the correct way in which experimental data in the oceanographic context should be interpreted.

In optics, on the other hand, both the dynamics and statistics of linear, nonlinear and hybrid focussing processes can be explored very conveniently to generate large and high quality data sets under a wide range of conditions. In addition, work in telecommunications aiming to propagate data signals over long distances in fibre optics networks since the 1990s has led to a number of very sophisticated approaches in studying noise propagation in nonlinear Schrödinger equation (NLSE) type systems [11-15]. Related recent work in optics has also seen the field of incoherent propagation effects becoming a subject of interest, with new techniques being used to analyze the appearance of long-term coherent structures from incoherent optical inputs [16]. It is the application of these recent advances in optics that may promise to shed new light onto the oceanic extreme wave counterparts.

\section{3 “Rogue Waves" in Nonlinear Fibre Optics}

\subsection{Supercontinuum Instabilities}

The field of nonlinear fibre optics is of course very mature, and fibre systems have been used to study wave mixing, modulation instability and soliton propagation since the early 1980's [3]. However, the development of the photonic crystal fibre (PCF) and the wider availability of new sources and components has revolutionised experimental fibre optics in recent years $[17,18]$.

In this context, a particular example is the process of fibre supercontinuum (SC) generation observed in PCF [19]. From its first observation in 1999-2000 [20], it has led to numerous important new applications, as well as motivating intense studies into the underlying theoretical description. The noise properties of the broadband SC spectrum have always been of significance for applications e.g. in frequency metrology [21], but until 2007, these were generally measured using only radiofrequency $(\mathrm{RF})$ techniques. The particular advance reported by Solli et al. in Ref. [2] was the use of ultrafast detection techniques to provide access to the shot-to-shot statistics of the pulse peak power, allowing the construction of a peak power histogram, and motivating the analogy with the long-tailed "L-shaped" histograms seen previously in oceanographic studies [5].

The presence of such extreme-value statistics in SC generation can be conveniently illustrated numerically, and simulated statistics of optical rogue waves were indeed carried out in parallel with the initial experimental studies [2]. Fig. 1 reproduces some of our own numerical simulations modeling SC generation under similar conditions, where the spectral broadening is seeded by modulation instability excited by a narrowband pump (in this case a 5 ps pulse) in the presence of broadband noise. The noise in the simulations is a semiclassical approximation to broadband quantum noise (a one photon per mode spectral density with random phase) but in practice many other noise sources in optics could contribute to seeding the instability (e.g. pump laser or optical amplifier noise). Further details of the modelling can be found in Ref [22].

The results in Fig.1 (a) superpose a large number of independently-generated output spectra (gray traces) from an ensemble of 1000 simulations obtained using different random noise seeds on the initial conditions. The black line is the mean spectrum. For our purposes, the key result from these simulations is the observation of significant shot-to-shot variation in the broadband $\mathrm{SC}$ spectrum that causes dramatic deviations from the mean. In fact, wavelength-dependent intensity fluctuations in SC generation had already been analysed to some extent in previous studies [23], but the originality of Solli et al. was to isolate the particular fluctuations on the long wavelength edge through the use of spectral filtering and fast detection of amplitude fluctuations. Note in this regard that later work showed that the particular measurement technique used by Solli et al. could be simplified because the same statistics could be seen even when using energy detection [24]. In addition, efforts continue to study the detailed way in which spectral filtering influences the statistical distributions observed and their interpretation (see the paper by Erkintalo et al. in this Special Issue).

The expanded view in Fig. 1(b) allows us to clearly see the small number of rogue "events" associated with a greatly increased red shift of the spectrum, and these particular events can 

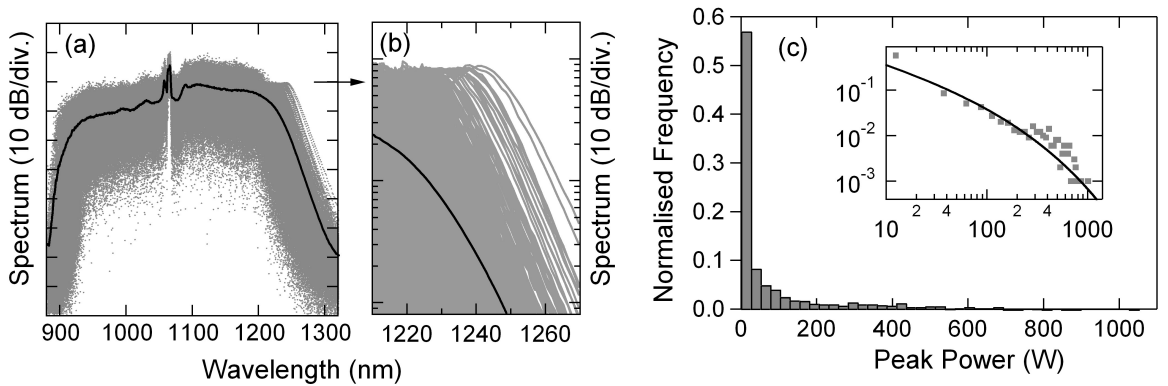

Fig. 1. a) Results showing 1000 individual spectra (gray curves). The mean spectrum is shown as the solid black line. (b) Expanded view above $1210 \mathrm{~nm}$. (c) Histogram of the peak power frequency distribution using $25 \mathrm{~W}$ bins. We plot normalized frequency such that bar height represents the proportion of data in each bin. The inset plots the results on a log-log scale, and also shows the fitted Weibull distribution (solid line). Ref. [22] provides further details of these simulations.

be isolated using a numerical procedure equivalent to that used in experiments. Specifically, for each SC in the ensemble, a filter selects components above a particular wavelength, and Fourier transformation yields a series of ultrashort pulses of varying peak power. The frequency distribution of the pulse peak power then readily reveals the presence of statistically-rare high peak-power events. Fig. 1(c) presents the data histogram, showing that the fraction of high power rogue events is extremely small. This can be seen more clearly on the log-log representation in the inset and indeed, the histogram can be shown to be well-fitted by a Weibull distribution, one that is commonly used to analyze extreme value events. This fit is shown as the solid line in Fig. 1(c).

The initial interpretation of these long wavelength events as a class of optical "rogue waves" was made on the basis of several factors. Firstly, there is a clear qualitative correspondence between the long tailed nature of the histogram in the figure and similar histograms seen in studies of rogue wave height distributions in oceanography. Secondly, many previous studies of nonlinear waveshaping mechanisms in oceanography were known to be based on NLSE (or extended NLSE) propagation models, and the role of MI had been considered in detail as a contributing mechanism to ocean wave formation (see for example Ref. [5], Chapter 4). In these optical experiments it is the input noise on ultrashort laser pulses injected into an optical fibre that seeds the development of an unstable modulation on the pulse envelope that evolves with propagation and eventually generates a small number of extremely large amplitude waves. Closer inspection of the particular events associated with the long tail of the histogram in the optical experiments identified clearly that the filtering process yielding the long tailed statistics was actually spectrally selecting localised optical soliton pulses emerging from the MI phase of the SC generation and undergoing particularly extended long wavelength shifts from intrapulse Raman scattering. These events were therefore described in Ref. [22] as "rogue solitons".

On the other hand, directly identifying these particular structures with oceanic rogue waves raises many questions, because the propagation of coherent solitons over distances long enough to undergo significant frequency downshifting is difficult to envisage in the noisy environment of the ocean. Nonetheless, the initial interpretation to link fibre instabilities to the oceanic rogue waves was highly imaginative and insightful, and motivated much subsequent work to identify where, if at all, the correct correspondence between fibre and oceanic rogue waves could be made. Interestingly, it has been shown that the emergence of such extreme events is not restricted to the original case investigated in the Nature 2007 paper with picosecond excitation but has also been shown to occur for a wider scale of pump pulse durations ranging from femtosecond pulses [25] to continuous wave pumps [26]. In fact, the only pre-requisite for observation of optical rogue waves in SC generation is an initial stage of noise-seeded modulation instability. But of course, it can be envisaged that any noise-driven nonlinear process might lead to the occurrence of extreme events in an optical context. 
The observation of extreme-value events in fiber-optic systems has also led to the question whether these can be controlled or triggered in some way upon seeding. A detailed study of the influence on the SC spectrum of a weak (short) seed injected together with a strong (long) pump has shown the broadening dynamics and statistics can be significantly influenced by the relative pump-seed time delay and frequency shift [27]. Other work has made connections with ideas from the domain of "induced MI" via imposing a small modulation across the full envelope of an input pulse, showing that the emergence probability of optical rogue solitons can be significantly enhanced [22]. This approach was further developed to show that if the input modulation parameters are carefully selected so as to suppress the modulation instability phase and seed preferentially the development of higher-order solitons, it appears possible to obtain the generation of a fully coherent SC from an arbitrary long pulse [28]. An interesting direction for future research here is perhaps to see how these studies of SC seeding and stabilisation may relate to earlier work on fluctuations and noise in amplifier and laser dynamics ("coherent photon seeding" or Hopf-Overman "phase wave" propagation) [29-32].

\subsection{Emergent Breathers}

Whatever the exact relation between solitons observed on the long wavelength edge of a broadband SC spectrum and large amplitude waves on the ocean, if an optical system is to be used as an analogue to study oceanic wave propagation, then it is important to clarify under what conditions the analogy is appropriate.

To this end, a series of recent papers by Akhmediev and co-workers have pointed out that a additional (and perhaps more likely) analogy with the oceanic rogue wave case is to be found in the onset phase of optical supercontinuum generation when coherent "breather" structures first begin to emerge [33-37]. Significantly, it has also recently been realised that the spectral characteristics seen in this emergent SC regime can be described using powerful analytic techniques based on the theory of a particular class of "Akhmediev Breather" (AB) structure [38]. Interestingly, despite intensive research over three decades, there are surprisingly-few analytic solutions describing nonlinear pulse evolution in optical fibers. Certainly there are exceptions such as the well-known soliton and self-similar solutions [39], but complex processes such as supercontinuum generation have generally been considered to require numerical approaches. These recent papers, however, have shown that the theory of AB evolution developed over 20 years ago can indeed successfully describe the form of the developing SC spectrum under long-pulse excitation conditions [40]. This provides new insight into the properties of the initial broadening and nonlinear spectral transformation of CW supercontinuum generation seeded by noise by showing how the temporal structure that develops from spontaneous MI can be naturally interpreted in terms of the characteristics of AB pulses. This work is very promising in identifying a particular regime of nonlinear propagation in optics where useful links with oceanic wave shaping may be found.

In this regard, we note that further research is still needed in order to clarify the way in which the amplitude of breather pulses can exhibit the characteristic long-tailed statistics of the oceanic wave case, but some results in this direction have recently been obtained [34]. Other recent research has focussed on the role of collisions due to symmetry breaking perturbations in breather propagation in leading to energy accumulation and localisation [41,42]. Numerical results adapted from Ref. [42] are shown in Fig. 2 where we compare (a) spontaneous breather formation dynamics from MI (a) in an NLSE system and (b) in an NLSE system modified by third-order dispersion. Because of the effects of noise, the drift in the breather propagation introduced by the dispersive perturbation does not occur uniformly across the temporal extent of the field and it is this that leads to collisions and the formation of localised pulses; closer analysis reveals that it is these pulses that subsequently evolve into the distinct rogue soliton pulses of Solli et al. Also, when discussing collisions, we stress that the although these recent papers have analysed collision processes in detail, the importance of collisions in the dynamics of optical rogue wave formation was identified earlier in Ref. [43]. Of course the role of collisions in SC generation was identified much earlier still $[44,45]$. 
(a) NLSE with noise

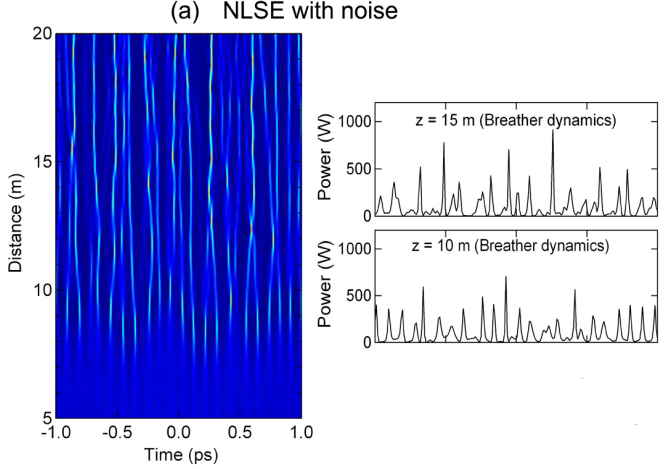

(b) NLSE with TOD and noise

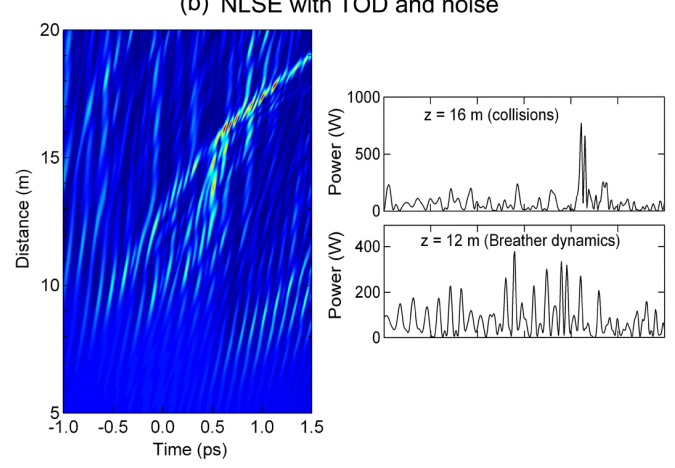

Fig. 2. (a) Detailed view of propagation in the NLSE with noise including line profiles of intensity at propagation distances as indicated. (b) shows the evolution for the case with the effect of TOD. Both figures show initial evolution associated with noise-induced MI and the appearance of high-contrast pulses. When TOD is included in the simulations, there is an additional phase of drift, interactions, collisions and localisation towards the appearance of solitons. See Ref. [42] for further details.

Finally in this section, we also note that aspects of these collision processes have also been related to earlier ideas where soliton turbulence was applied as a description of the nonlinear interactions that can occur in a perturbed NLSE type system[46]. Recent work applying kinetic theory to explain certain features of the supercontinuum spectrum is also noteworthy and provides a further example of how ideas from different areas of physics are being found increasingly useful in this field [47].

\subsection{Statistical Issues}

Analysing the statistics of any particular nonlinear propagation effect that yields localisation is of course essential in discussing the "rogue" or "extreme-value" properties rigorously. To this end, we comment here on possible future research directions in this particular area of the field, disussing some particular links with previous optics research that may prove fruitful to consider in more detail.

The events or configurations that lead to rogue waves are by definition extremely rare. As a result, the realistic modelling of rogue waves presents a fundamental mathematical challenge. Analytical methods alone cannot hope to deal with the complexity of the real system. Approximations can certainly be used to make analytical approaches possible, but it is impossible to tell if these approximations fatally impair the accuracy of the model. Computational methods may possibly handle the complexity of the systems, but on their own they have little hope of dealing with the rare events, particularly if there is a large parameter space that must be explored.

Recent work on stochastic methods applied to optical communication systems has shown that hybrid analytical/computational techniques have the potential of bypassing the difficulties encountered when each method is used separately. In particular, reduced analytical models, i.e., approximate solutions of the governing equations obtained with perturbation methods, linearization, or asymptotic techniques, combined with the application of variance reduction techniques such as importance sampling and other related methods, can provide accurate information about rare events at probability levels that are orders of magnitude beyond results obtained with traditional methods. The idea behind these methods is to use reduced models, not for the direct construction of approximate solutions, but rather to guide the biasing of full numerical solutions. The advantage of this approach is that numerical simulations, which involve no approximations and are therefore fully accurate, can specifically target only the most important regions of the probabilistic state space [11,48-51]. 
When a sufficiently accurate reduced or approximate model is not immediately available, it is also possible to use iterative and/or adaptive methods for the study of rare events. One such method is the multicanonical Monte-Carlo method which can be viewed as an example of a Markov chain Monte-Carlo method, where random samples are generated using a Markov process. Another promising method is the interacting particle system proposed in Ref. [52], which can be considered as a class of Monte-Carlo acceptance/rejection methods. Both these techniques have already been applied successfully to problems studying performance limitations in optical communications systems and should be very well-suited to a more rigorous analysis of the statistical properties of rogue wave events in optics [52-54].

\section{Long-Tailed Statistics in Other Optical Systems}

The 2007 experiments of Solli et al. have attracted considerable attention since their publication. Much of this initial impact was certainly due to the exciting possibility to apply a convenient fibre-based system to study the wider problem of oceanic instabilities, but there was also realisation that the results obtained represented a significant advance in characterising noise processes in a purely optical context.

The possibility to use a histogram to characterise the statistical properties of a nonlinear process has now motivated research in many other related fields of photonics research. In fact, non-gaussian statistics in fibre optics had been studied earlier in the context of multichannel fibre communications [55-61] but the recent studies in optics that have focussed on long-tailed statistics have considered a very diverse range of systems including Raman amplification in silicon [62], fibre Raman and parametric amplifiers [63,64], optical filamentation [65] and localisation effects in liquid crystals [66]. In a sense the ubiquitous nature of long tailed statistics in nonlinear optics is not surprising because any nonlinear transfer function will transform gaussian noise into a non-gaussian distribution, but the importance and possible significance of events in the "long tail" of these distributions seems not to have been appreciated until now.

But an important point needs to be made here. It is perhaps not useful to refer to all "long tailed" distributions in optics as "optical rogue waves", and we suggest that this term be restricted to localised structures in NLSE-like systems only (rogue solitons, breathers etc) where a correspondence with the oceanic case is most justified.Other classes of long-tailed instability in optics lose nothing in their general interest being referred to as "extreme-value" processes which is in our view a more accurate description.

\section{Conclusions}

Our purpose in this paper has not been to provide a comprehensive review of either the field of optical or hydrodynamic rogue wave physics. Rather, our intention has been to provide a brief overview of a number of particular research directions that may be of potential interest in the future. A particular advantage of studying rogue wave like processes using optical systems is the fact that the high repetition rate of optical sources allows the convenient generation of large data sets, even for the long-tailed probability distributions expected of extreme value phenomena where the events under study occur with extremely low probabilities. Moreover, it is also possible to envisage more sophisticated fibre- based experimental setups that will allow other rogue wave generation scenarios to be explored. Experiments in optics therefore appear easier to implement and to analyse, but it is important to consider to what degree conclusions from studying localised optical pulses can be transferred to studying water waves. This is perhaps the single most important question to answer when assessing the more general impact of the field of optical rogue wave physics.

We thank the French Agence Nationale de la Recherche project MANUREVA (ANR-08SYSC-019) for support. Additional thanks are extended to the Academy of Finland (GG, Research grants \# 121953, \# 130099, and \# 1332279) the Institut Universitaire de France (JMD). Aspects of this work have been performed in the framework of the Research Networks GDR 
Phonomi2, and COST actions 299 FIDES and MP0702. It is a pleasure to thank N. Akhmediev, O. Bang, C. M. DeSterke, B. J. Eggleton, M. Erkintalo, K. Hammani, B. Kibler, P.-A. Lacourt and A. Picozzi for their enthusiastic participation and collaboration.

\section{References}

1. E. Castillo, A.S. Hadi, N. Balakrishnan, J.M. Sarabia, Extreme Value and Related Models with Applications in Engineering and Science (Wiley, 2005)

2. D.R. Solli, C. Ropers, P. Koonath, B. Jalali, Nature 450, 1054 (2007)

3. G.P. Agrawal, Nonlinear Fiber Optics, 4th edn. (Academic Press, San Diego, 2006)

4. M. Olagnon, M. Prevosto, eds., Rogue Waves 2008, Brest, France 13-15 October 2008 (2008)

5. E. Pelinovsky, C. Kharif, eds., Extreme Ocean Waves (Springer, Berlin, 2008)

6. C. Kharif, E. Pelinovsky, A. Slunyaev, Rogue Waves In The Ocean, Advances in Geophysical and Environmental Mechanics and Mathematics (Springer, Berlin, 2009)

7. V.I. Bespalov, V.I. Talanov, JETP Letl. 3, 307 (1966)

8. V.E. Zakharov, L.A. Ostrovsky, Physica D 238, 540 (2009)

9. V.E. Zakharov, A.I. Dyachenko, A.O. Prokofiev, Eur. J. Mech. B/Fluids 25, 677692 (2006)

10. H. Tamura, T. Waseda, Y. Miyazawa, Geophys. Res. Lett. 36, L01607 (2009)

11. J.C. Chen, D.Q. Lu, J.S. Sadowsky, K. Yao, IEEE J. Sel. Areas Commun. 11, 289299 (1993)

12. G.E. Falkovich, I. Kolokolov, V. Lebedev, S.K. Turitsyn, Phys. Rev. E 63, 025601 (2001)

13. A.R. Holzlohner, C. Menyuk, Opt. Lett. 28, 1894 (2003)

14. J. Garnier, P. Del Moral, Opt. Commun. 267, 205 (2006)

15. R.O. Moore, G. Biondini, W.L. Kath, SIAM J. Appl. Math. 66, 1418 (2007)

16. A. Picozzi, Opt. Express 15, 9063 (2007)

17. P. St. J. Russell, IEEE J. Lightwave Technol. 24, 4729 (2006)

18. J.M. Dudley, J.R. Taylor, Nat. Photon. 3, 85 (2009)

19. J.M. Dudley, G. Genty, S. Coen, Rev. Mod. Phys. 78, 1135 (2006)

20. J.K. Ranka, R.S. Windeler, A.J. Stentz, Opt. Lett. 25(1), 25 (2000)

21. K.L. Corwin, N.R. Newbury, J.M. Dudley, S. Coen, S.A. Diddams, K. Weber, R.S. Windeler, Phys. Rev. Lett. 90, 113904 (2003)

22. J.M. Dudley, G. Genty, B.J. Eggleton, Opt. Express 16, 3644 (2008)

23. K.L. Corwin, N.R. Newbury, J.M. Dudley, S. Coen, S.A. Diddams, B.R. Washburn, K. Weber, R.S. Windeler, Appl. Phys. B 77, 269 (2003)

24. C. Lafargue, J. Bolger, G. Genty, F. Dias, J.M. Dudley, B.J. Eggleton, Electron. Lett. 45, 217 (2009)

25. M. Erkintalo, G. Genty, J.M. Dudley, Opt. Lett. 34, 2468 (2009)

26. A. Mussot, A. Kudlinski, M. Kolobov, E. Louvergneaux, M. Douay, M. Taki, Opt. Express 17, $17010(2009)$

27. D.R. Solli, C. Ropers, B. Jalali, Phys. Rev. Lett. 101(23) (2008)

28. G. Genty, J.M. Dudley, IEEE J. Quantum Electron. 45, 1331 (2009)

29. F.A. Hopf, M.A. Overman, Phys. Rev. A 19, 1180 (1979)

30. P. Beaud, J. Bi, Q. Hodel, H.P. Weber, Opt. Commun. 80, 31 (1990)

31. P.D. Drummond, J.D. Harvey, J.M. Dudley, D.B. Hirst, S.J. Carter, Phys. Rev. Lett. 78(5), 836 (1997)

32. G. New, M. Noy, J. Crosse, A. Rumley, L. Newson, Z.Y. Chen, C. Cheung, A. Todhunter, Opt. Commun. 282, 4418 (2009)

33. N. Akhmediev, A. Ankiewicz, M. Taki, Phys. Lett. A 373, 675 (2009)

34. N. Akhmediev, J.M. Soto-Crespo, A. Ankiewicz, Phys. Lett. A 373, 2137 (2009)

35. A. Ankiewicz, N. Devine, N. Akhmediev, Phys. Lett. A 373, 3997 (2009)

36. N. Akhmediev, A. Ankiewicz, J.M. Soto-Crespo, Phys. Rev. E 80, 026601 (2009)

37. N. Akhmediev, J.M. Soto-Crespo, A. Ankiewicz, Phys. Rev. A 80, 043818 (2009)

38. J.M. Dudley, G. Genty, F. Dias, B. Kibler, N. Akhmediev, Opt. Express 17, 21497 (2009)

39. J.M. Dudley, C. Finot, D.J. Richardson, G. Millot, Nature Physics 3, 597 (2007)

40. N. Akhmediev, V.I. Korneev, Theor. Math. Phys. 69, 1089 (1986)

41. M. Taki, A. Mussot, A. Kudlinski, E. Louvergneaux, M. Kolobov, M. Douay, Phys. Lett. A 374, $691(2010)$

42. G. Genty, C. de Sterke, O. Bang, F. Dias, N. Akhmediev, J. Dudley, Phys. Lett. A 374, 989 (2010) 
43. G. Genty, J.M. Dudley, B.J. Eggleton, Appl. Phys. B 94, 187 (2009), original publication: arXiv:0809.2388v1, 14 Sept. 2008

44. M.H. Frosz, O. Bang, A. Bjarklev, Opt. Express 14(20), 9391 (2006)

45. F. Luan, D.V. Skryabin, A.V. Yulin, J.C. Knight, Opt. Express 14(21), 9844 (2006)

46. V.E. Zakharov, N. Pushkarev, V.F. Shvets, V.V. Yan'kov, JETP Lett. 48, 83 (1988)

47. A. Picozzi, B. Barviau, B. Kibler, S. Rica, Eur. Phys. J. Special Topics 173, 297 (2009)

48. G.S. Fishman, Monte Carlo: Concepts, Algorithms, and Applications (Springer, New York, 1995)

49. P.J. Smith, M. Shafi, H.S. Gao, IEEE J. Sel. Areas Commun. 15, 597613 (1997)

50. A. Owen, Y. Zhou, J. Amer. Stat. Assoc. 95, 135 (2000)

51. G. Biondini, W. Kath, C. Menyuk, IEEE J. Lightwave Technol. 22, 1201 (2004)

52. P. Del Moral, J. Garnier, Ann. Appl. Probab. 15, 2496 (2005)

53. D. Yevick, IEEE Photon. Technol. Lett. 14, 1512 (2002)

54. D. Yevick, IEEE Photon. Technol. Lett. 15, 224 (2003)

55. C.R. Menyuk, Opt. Lett. 20, 285 (1995)

56. F.K. Abdullaev, S.A. Darmanyan, F. Lederer, Opt. Commun. 126, 89 (1996)

57. T. Georges, Opt. Commun. 123, 617 (1996)

58. G.E. Falkovich, M.G. Stepanov, S.K. Turitsyn, Phys. Rev. E 64, 067602 (2001)

59. S.A. Derevyanko, S.K. Turitsyn, D.A. Yakushev, Opt. Lett. 28, 2097 (2003)

60. Y.J. Chung, A. Peleg, Nonlinearity 18, 1555 (2005)

61. A. Peleg, Phys. Lett. A 360, 533 (2007)

62. D. Bourlaug, S. Fathpour, B. Jalali, IEEE Photonics J. 1, 33 (2009)

63. K. Hammani, C. Finot, J.M. Dudley, G. Millot, Opt. Express 16, 16467 (2008)

64. K. Hammani, C. Finot, G. Millot, Opt. Lett. 34, 1138 (2009)

65. J. Kasparian, P. Bejot, J.P. Wolf, J.M. Dudley, Opt. Express 17(14), 12070 (2009)

66. A. Montina, U. Bortolozzo, S. Residori, F.T. Arecchi, Phys. Rev. Lett. 103, 173901 (2009) 